\title{
Performance of the upgraded LTP-II at the ALS Optical Metrology Laboratory
}

\author{
Jonathan L. Kirschman, Edward E. Domning, Wayne R. McKinney, \\ Gregory Y. Morrison, Brian V. Smith, and Valeriy V. Yashchuk* \\ Advanced Light Source, Lawrence Berkeley National Laboratory, Berkeley, California, 94720
}

\begin{abstract}
The next generation of synchrotrons and free electron laser facilities requires x-ray optical systems with extremely high performance, generally of diffraction limited quality. Fabrication and use of such optics requires adequate, highly accurate metrology and dedicated instrumentation. Previously, we suggested ways to improve the performance of the Long Trace Profiler (LTP), a slope measuring instrument widely used to characterize x-ray optics at long spatial wavelengths. The main way is use of a CCD detector and corresponding technique for calibration of photo-response non-uniformity [J. L. Kirschman, et al., Proceedings of SPIE 6704, 67040J (2007)]. The present work focuses on the performance and characteristics of the upgraded LTP-II at the ALS Optical Metrology Laboratory. This includes a review of the overall aspects of the design, control system, the movement and measurement regimes for the stage, and analysis of the performance by a slope measurement of a highly curved super-quality substrate with less than 0.3 microradian (rms) slope variation.
\end{abstract}

Keywords: slope measuring instrument, long trace profiler, LTP, 2D detector, systematic error reduction

\section{INTRODUCTION}

The next generation of synchrotrons and free electron laser facilities requires x-ray optical systems with extremely high performance, generally of diffraction limited quality. Fabrication and use of such optics requires adequate, highly accurate metrology and dedicated instrumentation. Previously, we suggested ways to improve the performance of the Long Trace Profiler (LTP), a slope measuring instrument widely used to characterize x-ray optics at long spatial wavelengths. ${ }^{1-3}$ Based on these suggestions, the long trace profiler, LTP-II, available at the Advanced Light Source (ALS) Optical Metrology Laboratory (OML) was recently upgraded in order to improve the reliability of the instrument and to provide the opportunity (due to a modern data acquisition system) for further development of a system with higher performance. The main result of the upgrade is the ability for reliable slope-measurement metrology of significantly curved x-ray optics (down to radii of 15-18 meters) with precision of about $0.25 \mu \mathrm{rad}(\mathrm{rms})$ or better. This precision has been demonstrated while measuring super polished spherical grating substrates for the MERLIN project. ${ }^{4}$ The precision is on the level of performance of the world best slope measuring instruments, such as BESSY NOM. ${ }^{5}$

A few years ago, such optics were not routinely available from industry as they are now. Moreover, the requirements for slope accuracy of the state-of-the-art x-ray optics are continuously escalating. Now optics with slope variation of $<0.1 \mu \mathrm{rad}(\mathrm{rms})$ are required, which is a vital need for new coherent x-ray light sources such as FEL facilities. ${ }^{6}$ At the same time, prospective focusing grazing incidence $\mathrm{x}$-ray optical elements are already designed to possess a characteristic curvature of about 10 meters and even smaller. Slope surface metrology of such optics requires a range of measurement by an order of magnitude larger of that what is available with existing instruments. In order to cover the range with stitching of multiple measurements, one needs an instrument with extremely high performance. ${ }^{7}$

In this article, we present an overview of research and development efforts at the ALS OML which allowed for recent improvement of LTP performance and provides an analysis of the limiting factors for the current ALS LTP-II. In order to provide the reader with a complete set of relevant information, we include a number of references to ALS Light Source Beamline (LSBL) Notes that are available from the authors upon a direct request. Nevertheless, the list of references is far from the complete in that we do not try to review all world-wide efforts in the same direction but rather concentrate on our own work.

*vvyashchuk@lbl.gov; tel +1-510-495-2592; fax +1-510-486-7696 
Section 2 provides a critical review of the LTP optical schematic and its strengths and weaknesses. This is based on work performed at the ALS OML to delineate the major sources of random and systematic errors of the LTP-II available at the ALS OML. The premises of the upgrade and performance improvements of the upgraded ALS LTP-II instrument are considered in Sec.3. Ways for further improvement of the ALS LTP-II instrument that from our point of view will be effective in reaching the goal of $0.1 \mu \mathrm{rad}(\mathrm{rms})$ accuracy of the LTP are discussed in the conclusion.

\section{REVIEW OF PERFORMANCE OF THE LONG TRACE PROFILER LTP-II}

\subsection{Basic principles of the LTP}

The long trace profiler available in the OML belongs to the second generation of the LTP systems; this is known as the LTP II. The LTP-II was designed as a basic metrology tool for high accuracy testing of the figure of x-ray optics with slope variations on the order of one $\mu \mathrm{rad}(\mathrm{rms}){ }^{8-13}$ The LTP optical schematic is a realization of the pencil-beam interferometer system initiated by K. von Bieren, ${ }^{14}$ and developed and introduced to the synchrotron radiation community by Peter Takacs. ${ }^{8-10}$ In the interferometer, two parallel light beams, possessing a phase difference of $\pi$, are made to interfere at the focus of the Fourier transform lens (FTL). The resulting interference fringe pattern recorded with a position-sensitive detector, linear photo-diode array, placed at the focus, has two strongly marked peaks with a minimum between the peaks. The position of the minimum is a measure of slope of the mirror surface at the place of the beam reflection. The LTP records the local slope profile of a surface by measuring the reflection angle of a laser sample beam as the beam is transported across the surface by an air bearing carriage. Due to the translation of the optical sensor, the LTP has the unique capability for surface figure metrology of very long, meter size, mirrors. ${ }^{15-17}$

However, non-idealities of the translation mechanism as well as non-idealities of the optical elements result in a number of systematic effects, which often dominate the random noise of the LTP measurement. ${ }^{18-20}$ Some of the systematic effects have been analyzed and methods for their suppression have been developed. ${ }^{21-24}$

The main difference between an LTP II and an instrument of the first generation is the addition of a reference arm. ${ }^{12,13}$ In the optical schematic of this instrument, the reference slope signal from a stationary reference mirror is recorded simultaneously with the slope trace measurement of a mirror under investigation. Then, the reference trace is subtracted from the sample trace, providing data basically free of errors from wobbling of the LTP carriage and pointing instability of the laser beam. A similar approach has been used in a dual-beam laser deflection sensor described in Ref. ${ }^{25}$ In the original suggestion, ${ }^{12,13}$ the reference channel did not have the Dove prism, which was added later ${ }^{21}$ in order to combine with correct signs the errors due to the carriage wobbling and due to the laser pointing instability. The scale of angles of wobbling of the ALS LTP-II carriage accounted this way is about $10 \mu \mathrm{rad}$ that is determined by the figure error of the instrumental ceramic beam. Long term pointing instability (for an hour required for a scan with 1-meter long optics) of the current LTP diode laser (that has no temperature stabilization) is on the same level. Thus, due to the optical reference channel, profilers of the LTP-II type became capable of slope measurements with accuracy of about $1 \mu \mathrm{rad}$.

Paradoxically, the further improvement of the LTP performance to the level of modern requirements of $\sim 0.1 \mu$ rad must overcome a number of disadvantages inherent to the optical reference channel. First, three additional optical elements (Dove prism, reference mirror, quarter-wave plate) are potentially the sources for systematic errors due to the imperfections of their materials and fabrication. Second, the optical path of the reference beam, in general, changes while the LTP optical head is translated. As a result, the noise and systematic errors due to the imperfections of the LTP optics and convection of air along the optical paths also change. Moreover, when sufficiently long mirrors are measured, the reference optical path becomes so long that the reference beam comes too close to the edge of the apertures of the LTP optics. This leads to the noticeable perturbation of the reference interference pattern and, therefore, to the associated error of positioning of the pattern. Third, the optical paths for the reference and the sample beams are forced to be significantly different in order to avoid overlapping of the corresponding interference pictures (the 'ghost' effect ${ }^{23}$ ) on the detector. Besides, a significant difference of the random noise due to air convection, ${ }^{26}$ leads to the different systematic errors in the sample and reference channels associated with imperfections of the LTP optical elements. Therefore, the systematic errors can not be completely eliminated by the subtraction of the reference signal from the sample signal.

It should be mentioned that alternative schematics for a slope measuring instrument are possible with using a movable pentaprism and stationary optical head that is an LTP-like head as in Ref. ${ }^{15}$ or an autocollimator for precise angle measurement, as it is in the NOM. ${ }^{5}$ However, in spite of the fact that such an instrument has no reference channel, it is still subject to the errors associated with a changing optical path in the course of measurement. Moreover, because of the 
strong dependence of calibration on the distance between the reflective surface and the stationary sensor head (autocollimator), ${ }^{27,28}$ significant efforts to precisely calibrate the instrument are required. Note that generally the optical reference channel of an LTP-II profiler requires a similar sophisticated calibration that would be capable of accounting for the change of the carriage position with respect to the reference mirror. ${ }^{29,30}$

\subsection{Major sources of random noise of LTP measurements}

The sources and magnitudes of the LTP-II random noise are summarized in Table 1. The values of noise in the first two lines of Table 1 are found by subtraction of two sequentially measured traces in the corresponding channel. In this way, the systematic (repeatable) part of the traces is removed; and the result is random noise of a single measurement reduced by a factor of $\sqrt{2}$. The values in the table are renormalized in order to correspond to the single measurement random noise. Note that the procedure used here also removes a systematic variation of the reference slope traces due to figure error of the LTP ceramic beam.

The value in the third line of Table 1 corresponds to the random noise of a single measurement that is synchronous (correlated) in both the sample and reference channels. In the resulting slope trace obtained after subtraction of the reference trace from the sample one, this part of random noise is efficiently eliminated. Additional measurements performed with the LTP have allowed separating the contributions of carriage wobbling and laser beam pointing instability to the eliminated part of the noise. The corresponding values are shown in the 4th and 5th lines of Table 1, respectively. The next two lines in the table provide the estimated values of random noise due to the LTP-II detector and the lab environmental conditions.

The last line in Table 1 presents an experimentally found value of random noise of a single LTP measurement performed in the stationary (no carriage translation) mode. The result, about $0.7 \mu \mathrm{rad}(\mathrm{rms})$, is similar to that obtained while measuring over the clear aperture of $120 \mathrm{~mm}$ of a $28-\mathrm{m}$ spherical mirror. In this case, the value of the random noise was obtained by subtraction from a single measurement a trace averaged over multiple repeatable measurements, where the random contribution into the resulted slope trace is significantly suppressed.

In Ref. ${ }^{26}$ it has been shown that the random noise of the LTP is mainly due to air-convection (turbulence) along the optical paths of the reference and sample laser beams. This is a low frequency $(<1 \mathrm{~Hz})$ noise that is very difficult to average. In order to average the noise of $0.67 \mu \mathrm{rad}$ (rms) to the level of $\sim 0.1 \mu \mathrm{rad}$ (rms) (that corresponds to the random noise of the LTP-II detector), one should perform at least 45 measurements. Another solution has been suggested: ${ }^{26}$ apply air blowing in order to shift the noise frequency range to higher frequency, more suitable for effective averaging.

\subsection{Major sources of systematic errors of LTP measurements}

Nevertheless, performance of the LTP-II is still limited by systematic errors rather than random noise. We assume here that the systematic errors due to profile imperfections of the ceramic beam and due to the pointing instability of the laser

Table 1. Performance of the LTP-II with photo-detector based on linear photo-diode arrays. The values of random noise are given for a single measurement with a $28-\mathrm{m}$ spherical mirror and with clear aperture of $120 \mathrm{~mm}$.

\begin{tabular}{|c|c|c|c|}
\hline Random Noise & rms value $[\mu \mathrm{rad}]$ & Comments & Refs. \\
\hline $\begin{array}{r}\text { Total in the } \\
\text { sample channel }\end{array}$ & 0.43 & $\begin{array}{l}\text { Experimentally found with subtraction of two consequently } \\
\text { measured sample traces }\end{array}$ & {$[30]$} \\
\hline $\begin{array}{r}\text { Total in the reference } \\
\text { channel }\end{array}$ & 0.7 & $\begin{array}{l}\text { Experimentally found with subtraction of two consequently } \\
\text { measured reference traces }\end{array}$ & {$[30]$} \\
\hline $\begin{array}{l}\text { Random wobbling } \\
\text { and laser pointing } \\
\text { instability }\end{array}$ & 0.54 & $\begin{array}{l}\text { Measured as a difference of the sum of the total noise in } \\
\text { reference and sample channels and the measured total random } \\
\text { noise of a single LTP measurement }\end{array}$ & {$[30]$} \\
\hline $\begin{array}{l}\text { Laser pointing } \\
\text { instability }\end{array}$ & 0.26 & $\begin{array}{l}\text { Measured with absolutely quiet laboratory conditions and } \\
\text { verified with direct measurement with a beam profiler }\end{array}$ & {$[30,31]$} \\
\hline $\begin{array}{l}\text { Random carriage } \\
\text { wobbling }\end{array}$ & 0.47 & $\begin{array}{l}\text { Significant non-uniformity of carriage motion (horizontal } \\
\text { accelerations) has been also observed }\end{array}$ & {$[30]$} \\
\hline $\begin{array}{l}\text { Random noise of the } \\
\text { detector }\end{array}$ & 0.1 & Estimated based on the detector parameters & {$[1,30,32,33]$} \\
\hline $\begin{array}{l}\text { Vibration, electrical } \\
\text { noise, etc }\end{array}$ & 0.1 & $\begin{array}{l}\text { Estimated from measurement with absolutely quiet laboratory } \\
\text { conditions }\end{array}$ & {$[1,30,33]$} \\
\hline Total: & $\approx 0.67$ & $\begin{array}{l}\text { Measured in stationary mode; reference channel allows } \\
\text { accounting of random noise due to the laser pointing instability }\end{array}$ & {$[30]$} \\
\hline
\end{tabular}


Table 2. Performance of the LTP-II with photo-detector based on linear photo-diode arrays. The values of noise are given for a single measurement with a 28-m spherical mirror and with clear aperture of $120 \mathrm{~mm}$.

\begin{tabular}{|l|c|l|c|}
\hline \multicolumn{1}{|c|}{ Systematic Error } & rms value $[\mu \mathrm{rad}]$ & \multicolumn{1}{c|}{ Comments } & Refs. \\
\hline PDA photo-response (at 2\%) & 0.3 & Estimated based on the manufacturing specification & {$[1,32]$} \\
\hline $\begin{array}{l}\text { PDA pixel pitch non- } \\
\text { uniformity (at 10\%) }\end{array}$ & 0.3 & Estimated based on the CMOS process tolerance & {$[1,32]$} \\
\hline $\begin{array}{l}\text { Imperfection of the LTP } \\
\text { optical elements }\end{array}$ & $0.2-0.9$ & $\begin{array}{l}\text { The error strongly depends on curvature of a mirror under } \\
\text { test and its distance from the LTP optical head }\end{array}$ & {$[27,29,34]$} \\
\hline Detector interference effect & $<0.03$ & Limit for 0.1 ${ }^{\circ} \mathrm{C}$ temperature instability during a run & {$[32]$} \\
\hline Detector dark signal & $\sim 0.01$ & Measured with the LTP-II & {$[32]$} \\
\hline Total: & $0.5-1.0$ & Estimated by summing the squared errors & \\
\hline
\end{tabular}

are totally accounted for with the LTP-II optical reference channel. Unfortunately, the reference channel in principle does not help much against the other major sources of the LTP systematic errors (i) non-ideality of the photodetector $^{1,33,34}$ and (ii) imperfections of the LTP optical elements ${ }^{30}$ - Table 2.

(i) The existing technologies for fabrication of PDA and CCD chips provide rather poor, $\sim 2 \%$, pixel-to-pixel uniformity of the photo-response. For the LTP-II PDA detector it leads to the systematic error of $\sim 0.3 \mu \mathrm{rad}$ (rms) due to the associated error of positioning of the interference pattern. ${ }^{1}$ We estimate the systematic errors via an rms variation of the measured trace due to the error. For the error related to the PDA photo-response non-uniformity, this is an rms variation of positioning for different places on the detector. $10 \%$ non-uniformity of the PDA pixel pitch also gives a systematic error of $\sim 0.3 \mu \mathrm{rad}(\mathrm{rms})$ - see the second line of Table 2 .

The systematic errors related to the LTP detector can be partially accounted via flat-field calibration of the photo-diode arrays. We have developed an efficient calibration technique based on a high power LED. ${ }^{2}$ The technique has been applied to calibrate the photo-response non-uniformity of the CCD camera detector of the upgraded ALS LTP-II with accuracy of $\sim 0.4 \%$ - see also the next section.

(ii) Imperfections (residual surface figure and non-homogeneity of optical materials) of the optical elements (the phase shifter, beam splitters, wave-plates, f-theta lens, folding and reference mirrors, Dove prism) can produce even larger systematic error as depicted with the third line of Table 2 . The value of the error strongly depends on the peculiarities of the optics under test (e.g., its surface shape) and experimental arrangement in the course of the measurement (e.g., positioning and alignment of the mirror in respect to the LTP optical head). ${ }^{28,30,35}$

Partially, the systematic errors due to the imperfections of the LTP optical elements can be reduced with repeatable measurements with different alignment and orientation of the optics under test. An additional possibility of such reduction appears if a 2D CCD camera is used as a detector ${ }^{36}$ (see also Sec. 3). In this case averaging of repeatable measurements performed at slightly different tilt in the sagittal direction of the mirror under test allows effective suppression of the low spatial frequency part of the systematic error.

Note that in Table 2, the total systematic error of 0.5-1.0 $\mu \mathrm{rad}(\mathrm{rms})$ is estimated for the LTP sample channels. The contribution of systematic errors to a completed LTP measurement can be larger.

\subsection{LTP drifts and instabilities}

Temperature gradients and drifts inside the LTP hutch cause drift and instabilities of the LTP components, as well as induce air convection along the light beam optical path and the associated random noise considered in Sec. 2.2. There are a few sources of the temperature variation and associated air convection.

First, the temperature in the OML periodically varies with amplitude of $\sim 120 \mathrm{mK}$ and a period of $\sim 12$ min corresponding to the switching on/off cycle of the lab air conditioner., ${ }^{3,37}$ In order to reduce temperature variation over the instruments, each metrology instrument in the OML is enclosed with a hutch. Typically, inside the LTP hutch the 12-min periodic temperature variation has amplitude of $\sim 5 \mathrm{mK}$. At first glance, that small variation would seem to be insignificant for the LTP performance. But it can be easily demonstrated e.g., with the ZYGO GPI interferometer ${ }^{38}$ that LTP performance on the level of $0.1 \mu \mathrm{rad}(\mathrm{rms})$ requires temperature stability of better than $\sim 1 \mathrm{mK}$ for the measurement time of an hour by visually observing fringe movement with small temperature variations. High temperature stability is a distinguishing property of the BESSY NOM set-up. ${ }^{5}$ The stability was made possible due to a combination of (i) a separation from a 
corridor with two buffer rooms, (ii) a high performance climatic system, (iii) a two-layer thermo-isolating hutch, (iv) a massive granite table, (v) absence of sources of vibration in the vicinity of the lab, and (vi) remote control measurement. Stable environmental conditions of the NOM allow for multiple repeated measurements for many hours without need for any readjustment or realignment of the optical elements. By averaging repeated measurements of the total number of 20 (a 4-5 hour run), the random noise of a slope trace measured with the NOM can be easily suppressed, down to the level of $\sim 0.05 \mu \mathrm{rad}$ (rms). This is an effective, but time consuming way to improve accuracy and reliability of surface slope measurements. Unfortunately, not all metrology labs are provided with resources for this approach.

Second, inside the LTP hutch, there are a number of sources of heat and air convection. These are the carriage driving motor, air flow of the air-bearing system, the detector, the laser and its power supplies, and the heat sinks and fans of the temperature stabilization systems of the LTP camera and tiltmeters. The effect of these sources of heat on an LTP measurement depends on the measurement peculiarities: position of the carriage, direction of translation of the carriage, measurement rate, etc. Variation of temperature of the LTP diode laser leads to a variation of wavelength of the emitted light and to a change of light beam direction (pointing) that is characterized with a parameter of pointing stability measured in units of $\left[\mathrm{rad} /{ }^{\circ} \mathrm{K}\right]$. At the usual experimental arrangement of the LTP, the drift of the beam direction can be as large as $5 \mu \mathrm{rad} /$ hour. To a significant extent but not totally (because of difference of the optical paths for the reference and the sample beams), the laser pointing instability of the LTP laser is compensated with the reference channel. Further improvement can be obtained by replacing the laser with a laser system less sensitive to the surrounding environmental conditions. We have experimentally investigated pointing instability of different lasers, ${ }^{32}$ including a diode laser similar to one used in the present version of the LTP, an intensity stabilized HeNe laser, and a fiber coupled, temperature stabilized diode laser. ${ }^{39}$ The measurements were performed with the NanoScan ${ }^{\mathrm{TM}}$ beam profiler ${ }^{40}$ capable of measuring a laser beam position with accuracy of $0.1 \mu \mathrm{m} / \sqrt{\mathrm{Hz}}$. For the LTP laser, the low frequency $(f \leq 1 \mathrm{~Hz})$ variations of laser pointing can be characterized with the rms variation measured (at quiet environmental conditions, similar to ones inside the LTP hutch) to be $\sim 0.3 \mu \mathrm{rad}-$ Table 1 . This parameter for the He-Ne laser is $\sim 0.5 \mu \mathrm{rad}$. The fiber coupled laser has demonstrated the best stability of $<0.1 \mu \mathrm{rad}$ (rms) that corresponds to the laser specification of $\sim 0.1 \mu \mathrm{rad} /{ }^{\circ} \mathrm{K}$. Such a fiber coupled diode laser would further improve the precision of the LTP measurements.

Beside the laser, the most temperature dependent element of the current ALS LTP-II is phase shifting system due to the temperature instability of the prism mounts. In Ref. ${ }^{41}$ different methods for obtaining the $\pi$-phase shift in a pencil-beam interferometer with a solid piece of glass have been considered. Such a shifter is less sensitive to temperature variation and should be incorporated in our LTP. However, there is question in principle what is the optimal phase shift value for an LTP. After answering this question, we will design a new shifter for the ALS LTP-II.

We should mention one more effect that is associated with a temperature drift of the laser wavelength. As it was shown in Ref. ${ }^{42}$ there is a dependence of the effective pixel optical center depending on the wavelength of the light. An effect of the order of $0.01 \mu \mathrm{m} / \mathrm{nm}$ (optical position varies by $\sim 30 \%$ of the pixel pitch between $905 \mathrm{~nm}$ and $400 \mathrm{~nm}$ ) was experimentally observed with a CCD with pixel pitch of $24 \mu \mathrm{m}$. The measurement of the variation of the optical position of the entire pixel requires a more sophisticated apparatus. At the current level of knowledge, we can only hope that the effect is not noticeable for the existing instruments and instrumental performance.

\section{PERFORMANCE OF THE UPGRADED ALS LTP-II INSTRUMENT}

The recent upgrade of the LTP-II at the ALS OML has included (i) re-assembling and re-adjustment of the LTP-II linear translation stage to allow assured operation under the National Instrument (NI) motion control system at increased loading due to the new detector; ${ }^{43}$ (ii) development of motion control and data acquisition software based on the NI LabView $^{\mathrm{TM}}$ platform; ${ }^{43,44}$ (iii) development of original software and methods for carrying out LTP measurements and data analysis; ${ }^{36,45}$ (iv) design and fabrication of a kinematical mount with active temperature stabilization for the CCD camera detector and a new camera calibration system and technique. ${ }^{2}$

\subsection{Ideas for the upgrade of the ALS LTP-II instrument}

The major ideas for the upgrade were considered in our previous work, ${ }^{1}$ where the LTP beam positioning error related to the detector was analyzed. It was shown that the error is significantly reduced if (1) size of a pixel of the detector is reduced; (2) precise calibration of the pixel-to-pixel photo-response non-uniformity is performed; and (3) a 2D CCD camera with 8 bit resolution is used rather than two 16 bit 1D PDA detectors. These brought up the major criteria for selection of a reliable 2D CCD camera to be used as a LTP detector. ${ }^{2,44}$ 
All major contributions to the positioning error related to the detector, such as read-out noise, photo-response nonuniformity, pixel pitch non-uniformity, depend dramatically on number of points (pixels) involved in finding the position (positioning) of the interference pattern minimum. Asymptotically, the dependence is inverse proportional to the square root of the number of points. Physically, the larger number of pixels provides more efficient averaging of all pixel-topixel non-uniformities, assuming that the non-uniformities have no strong spatial correlation. Note that the smaller pixel size provides a proportionally larger number of points for a PDA based detector, but a quadratically larger number of points for a 2D CCD camera based detector. This is one of the main reasons for use of a 2D square pixilated detector.

There are more advantages of use of a 2D detector. A 2D detector allows for simultaneously measuring the slope angle in two perpendicular directions. This is extremely important for accurate metrology of a twisted optics, as well as for precise realignment of sagittally curved optics.

Moreover, a 2D detector provides an excellent opportunity to use an efficient technique for suppression of the LTP systematic error. ${ }^{36}$ With a $2 \mathrm{D}$ detector, it becomes possible to perform repeatable measurements over the same trace on the mirror surface, but at different sagittal tilts of the mirror with respect to the LTP. Even at rather small sagittal tilt, about $50 \mu \mathrm{rad}$, the light beam path through the optical elements of the LTP significantly changes. This allows suppressing the LTP systematic error due to higher spatial frequency variations of properties of the LTP optics by averaging over such measurements. It should be mentioned that most of the active LTP instruments around the world use detectors based on linear arrays and, therefore, have no technical possibility for such suppression of LTP systematic errors. The only exclusion is the LTP at SOLEIL (France). ${ }^{46}$ However, even in this case, in order to suppress the optical systematic error, they tilt the surface undergoing metrology in the tangential direction. In this case, the measured traces are not absolutely independent, because the contributions of the systematic error are shifted along the measured trace, leaving the possibility for a correlation effect among the residual errors. Moreover, tilting in the tangential direction limits the slope measurement range of the instrument. However, combination of these two methods, with tilting in sagittal and tangential directions, can give an additional advantage. Note that the suggested method is applicable to the measurements with an autocollimator based profiler such as the BESSY NOM ${ }^{5}$ that has a 2D detector.

A 2D detector also allows the elimination from the LTP optical schematic a right angle prism, one of the LTP optical elements potentially contributing to the systematic error. The prism is used as a beam splitter to direct the sample and reference beams into the corresponding detector arm based on 1D photo-diode arrays.

In order to implement the techniques for suppression of instrumental nonlinear drifts, the motion control system of the upgraded LTP should provided the capability for multiple sequential scans with an arbitrary pattern of forward and reverse scans.

\subsection{The 2D detector of the upgraded ALS LTP-II}

Based on the consideration in the work, Ref. ${ }^{1}$ we have decided to use a Pantera SA 4M15 Area Scan Camera from DALSA Inc. ${ }^{48}$ The camera uses a $2048 \times 2048$ pixel sensor with $7.4 \times 7.4 \mu \mathrm{m} 2$ pixel size, capable of running at up to 16 frames per second, and features electronic global shuttering. The camera has programmable features and diagnostics accessible through the MDR26 Camera Link $^{\mathrm{TM}}$ control. The camera ADC provides true 8-bit digitization. The digitization on many cameras with a higher number of bits is often not accurate.

Figure 1 shows a model of the Pantera SA 4M15 camera assembly and a picture of the optical sensor of the upgraded ALS LTP-II with the new detector. The design of the camera mount allows for precisely positioning the camera sensor in the focal plane of the Fourier transform lens. The Peltier-element-based temperature stabilization system is capable of keeping the camera at approximately room temperature with $\sim 10 \mathrm{mK}$ stability at $6 \mathrm{~W}$ heating power. Temperature stabilization is essential for reliable use of the camera calibration data obtained in a dedicated experiment.

In order to get the best performance of the camera as a beam position sensor, the efficiency of each pixel has to be precisely calibrated. The calibration is necessary not only because of the pixel-to-pixel non-uniformity of the photoresponse discussed above. An additional problem can appear if a 2D camera has separated amplifiers for different subsets of pixels. For example, some interferometric microscopes have a camera with different amplifiers for odd and even pixel lines. In this case, absence of calibration dictates application of averaging over each two neighboring lines that, in turn, lead to loss of resolution in sagittal direction. ${ }^{48,49}$

The DALSA camera used for the upgraded LTP has different amplifiers for left and right halves of camera field of view. A difference in the gain factor of the amplifiers leads to a noticeable perturbation of a recorded image. In our application, it will lead to a systematic error of beam positioning. 


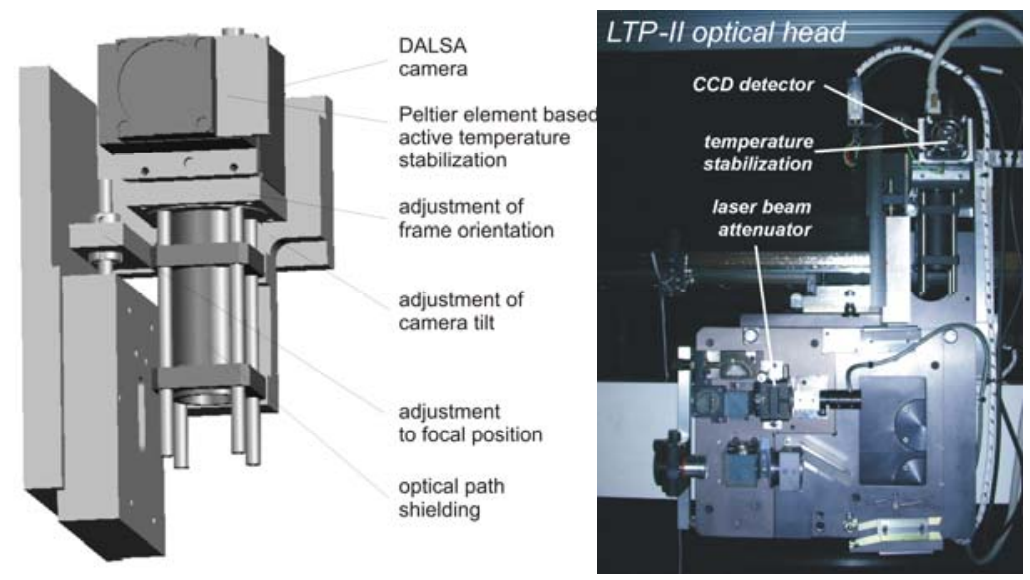

Figure 1: Model of the Pantera SA 4M15 camera assembly (the left-hand picture) and picture of the optical sensor of the ALS LTP-II upgrade with a new detector system based on the DALSA camera (the right-hand picture).

In order to precisely calibrate the camera, we need a flat field light source with uniformity of $\leq 0.5 \%$ over $15 \times 15 \mathrm{~mm}^{2}$ area that has an adequate dynamic range for the camera's 8-bit ADC. However, the commercially available flat-field light sources based on integrated spheres are generally specified to provide only $\sim 1 \%$ uniformity of the light across such an area. We have developed an original flat-field light source based on a high power LED with current and temperature stabilization. $^{2}$ It was measured to provide the uniformity of about $0.2 \%$, adequate to the 8 - and even 10 -bit ADC. We have performed an in-situ calibration with the camera mounted on the LTP as it is shown in Fig. 1. Note that there is not, in principle, a justification for using a camera with significantly higher resolution as an LTP detector. The only reason is to increase the camera dynamic range for light intensity measured. However, such a camera would require even more sophisticated (non-linear) calibration and will be significantly more expensive. We have easily solved the problem of the dynamic range with a simple attenuator based on a replaceable density filter and two crossed polarizers for fine adjusting the intensity of the laser light beam.

\subsection{Investigation of the performance of the upgraded ALS LTP-II}

The upgrade of the LTP-II system has significantly improved the reliability of the instrument. At the same time, the overall accuracy of the instrument generally remains the same, because all optical elements and the basic schematic of the upgraded LTP are still the same as they were in the original LTP-II.

Nevertheless, due to the sophisticated 2D detector, modified motional system, and a flexible data acquisition system, the limited precision of the instrument has been significantly improved and was estimated to be on the level of $0.25 \mu \mathrm{rad}$ (rms) when measuring a significantly curved ( $\sim 15 \mathrm{~m}$ radius) optic ${ }^{38}-$ Fig. 2 . Figure 2 verifies the $0.25 \mu \mathrm{rad}(\mathrm{rms})$ precision of metrology with the upgraded ALS LTP via a cross-comparison of the ALS metrology ${ }^{50}$ with BESSY measurements ${ }^{51}$ over the same areas of a $15 \mathrm{~m}$ curved Si super polished substrate for the MERLIN project - Fig. 2 . In general, the cross-check shows a good agreement of the measurements. However, there is also a noticeable discrepancy with magnitude up to $0.6 \mu \mathrm{rad}$. That is a clear indication of the LTP systematic errors, which we cannot totally avoid without a precise calibration of the instrument. Nevertheless, the result in Fig. 2 became possible only with the upgraded LTP-II when all developed methods and techniques are used.

The parasitic effects of the carriage wobbling and wiggling were minimized with the move-stop-wait-measure-move mode of measurement that becomes possible with the upgraded translation system for the LTP optical head based on LabView $^{\mathrm{TM}}$ motion control. ${ }^{43}$ A trade-off for this is a rather long time for measurement of a single point of about 8 sec. The main contribution to the time $(\sim 7 \mathrm{sec})$ comes from the feedback time of carriage positioning due to the use of two position sensors: a rotation encoder of the LTP motor and a distance measuring interferometer. ${ }^{43}$

With the high spatial resolution $2 \mathrm{D}$ camera with $7.4 \times 7.4 \mu \mathrm{m}^{2}$ pixel size (that has to be compared with $25 \mu \mathrm{m} \times 2.5 \mathrm{~mm}$ pixel size of the LTP-II PDA), and precise calibration of the photo-response and dark current for each pixel, we have decreased the error of positioning of an interference pattern to the level as low as 10 nrad. Note, that the developed positioning algorithm ${ }^{1}$ allows us to evaluate the error of positioning for each point of a slope trace; this is a new and very important feature of the updated LTP-II software. ${ }^{44,52}$ Ideally, a measured slope trace should have error bars corresponding to the error of the measured slope. The next version of the LTP analyzing software will provide such data. 


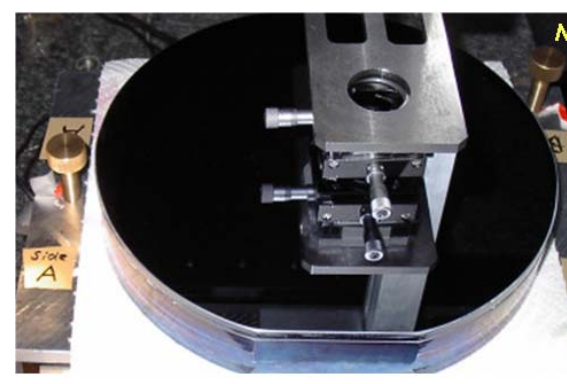

BESSY NOM:

Best fit radius: $\mathbf{1 4 . 9 6 2 0 \mathrm { m }}$ Slope Variation:1.44 $\mu \mathrm{rad}(\mathrm{pv})$ and $0.278 \mu \mathrm{rad}(\mathrm{rms})$

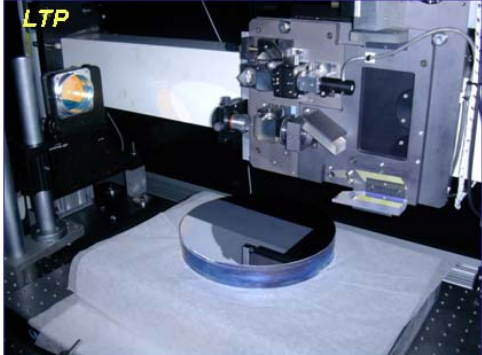

ALS LTP-ll:

Best fit radius: $15.065 \mathrm{~m}$

Slope Variation:1.44 $\mu \mathrm{rad}(\mathrm{pv})$ and $0.293 \mu \mathrm{rad}(\mathrm{rms})$

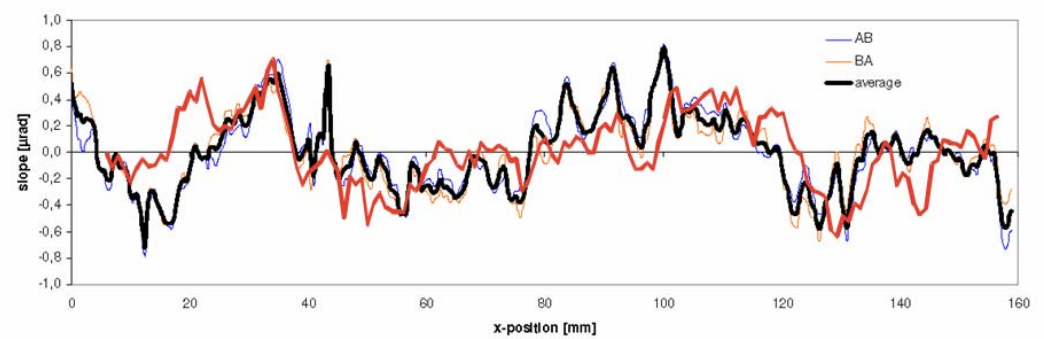

Figure 2: Metrology of 15-m spherical substrate for MERLIN project and comparison of the results obtained with the BESSY NOM ${ }^{51}$ and the upgraded ALS LTP-II. In the course of measurements at the ALS OML, three separately measured traces were stitched together. Each trace was obtained by averaging of sequential forward and reverse scans at six different sagittal tilts and at two opposite orientations of the substrate under test. The NOM picture and data are courtesy of Frank Siewert.

As discussed in Sec. 3.1, the 2D detector of the upgraded LTP provides an opportunity to significantly suppress the high spatial frequency part of the LTP systematic error ${ }^{36}$ by performing repeatable measurements over the same trace on the mirror surface, but at its different sagittal tilts.

Effective reduction of the air convection noise has become possible with averaging of multiple (we usually use 10) sequential camera frames measured with a uniform interval over a 1-sec total acquisition time. The 1-sec acquisition time is just adequate to suppress the characteristic noise power density spectrum of air convection measured inside the LTP hutch. ${ }^{26}$

Suppression of instrumental drift became possible with the upgraded translation system of the LTP. Now, measurements can be performed with carriage translation in forward and reverse directions in a straightforward manner. Averaging of two slope traces, obtained with the opposite translation directions, allows for effective suppression of a linear drift. With a more sophisticated pattern of forward and reverse measurements, one can suppress even higher order temporal drifts.

Table 3 summarizes the performances of the upgraded ALS LTP-II investigated by measuring super polished MERLIN substrates with radii of curvature of $\sim 18.5 \mathrm{~m}$ and $\sim 15.0 \mathrm{~m} .^{45,50}$

Table 3. Performance of the upgraded ALS LTP-II with 2D detector based on DALSA camera.

\begin{tabular}{|l|c|l|c|}
\hline \multicolumn{1}{|c|}{ Systematic Error } & rms value $[\mu \mathrm{rad}]$ & \multicolumn{1}{|c|}{ Comments } & Refs. \\
\hline $\begin{array}{l}\text { Overall error of } \\
\text { positioning }\end{array}$ & $\sim 0.01$ & $\begin{array}{l}\text { Calculated for each slope point in the course of fitting of } \\
\text { an interference feature }\end{array}$ & {$[1]$} \\
\hline $\begin{array}{l}\text { Unsuppressed } \\
\text { systematic error due to } \\
\text { imperfection of the LTP } \\
\text { optical elements }\end{array}$ & $\sim 0.25$ & $\begin{array}{l}\text { The error corresponds to the low spatial frequency } \\
\text { perturbation of the LTP optics that is impossible to } \\
\text { average by sagittially tilting a mirror under test }\end{array}$ & {$[35,37,44,49]$} \\
\hline Overall random error & $\sim 0.3$ & $\begin{array}{l}\text { Measured as a difference of the sum of the total noise in } \\
\text { reference and sample channels and the measured total } \\
\text { random noise of a single LTP measurement }\end{array}$ & {$[37,44]$} \\
\hline Total: & $\sim 0.4$ & $\begin{array}{l}\text { Estimated by summing the squared systematic and } \\
\text { random errors corresponding to a single slope trace }\end{array}$ & \\
\hline
\end{tabular}


The values of the errors in Table 3 are given for single measurement. With the known experimental precautions, the overall precision of a mirror slope metrology on the level of $0.25 \mu \mathrm{rad}(\mathrm{rms})$ became possible. By averaging over sequential measurements with different sagittal tilts of the optics under test, we have suppressed the higher spatial frequency systematic error. By additional averaging of traces obtained with the carriage translation in the forward and in the reverse directions, we have removed some systematic effects associated with linear drifts. By averaging over two sequential measurements with opposite orientations of the optics under measurement, the odd-symmetry part of the instrumental systematic error has been also removed. ${ }^{53}$

Figure 3 illustrates the irremovable systematic effect. In this case, measurements with the same optics were performed at different distances between the LTP optical head and the mirror surface. ${ }^{38}$ It is clearly seen in Fig. 3 that the residual slope variation is significantly larger at the larger distances, when the reflected beam is scanning across a larger aperture of the LTP optical elements, the pentaprism, and the Fourier transform lens.

From the above considerations, we conclude that we still need a sophisticated calibration system for precise calibration of the upgraded LTP and correction of the low-spatial-frequency systematic errors. The current calibration extracted from the test measurement with the $40 \mathrm{~m}$ curved Round Robin mirror ${ }^{45}$ and corrected via comparison of the measurements performed with the LTP and BESSY NOM over the $15 \mathrm{~m}$ MERLIN substrate, provides a one point calibration and does not account for the non-linearity of LTP measurements. A more sophisticated technique for calibration of slope measuring instruments is under development by a world-wide collaboration of the ALS, BESSY, and the PTB. ${ }^{28-30}$

\section{Grating blank measured with upgraded ALS LTP -II}

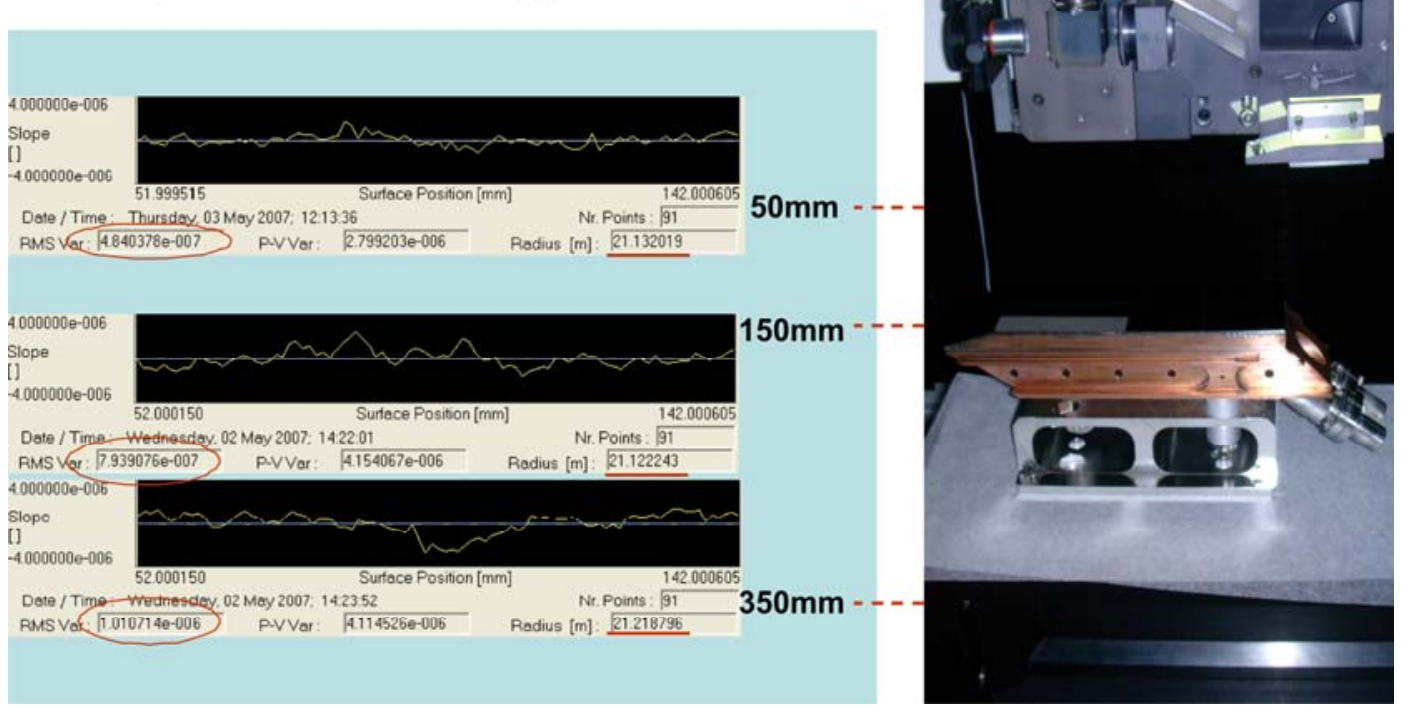

Figure 3: Metrology of 24-m spherical substrate at different distances between the measured surface and the LTP optical head.

\section{CONCLUSION}

In conclusion, we have reviewed the main problems of the LTP-II and discussed the most effective solutions used for the instrument upgrade.

In order to improve the accuracy of positioning of the LTP interference pattern, we developed a 2D detector system based on a DALSA camera with $2048 \times 2048$ pixels with $7.4 \mu \mathrm{m} \times 7.4 \mu \mathrm{m}$ pixel size. The camera was equipped with an active temperature stabilization system and was calibrated with a specially designed flat field light source. With a new fitting algorithm based on a regression method, we have evaluated the error of positioning to be as low as $10 \mathrm{nrad}$.

The new detector allows for efficient suppression of random noise due air convection by averaging over multiple (10) frames captured for total time of $\sim 1 \mathrm{sec}$. As a result, the random noise for a single measurement is about $0.3 \mu \mathrm{rad}(\mathrm{rms})$. 
We have developed a number of techniques that are shown to be very efficient for suppression of the instrumental systematic errors and drifts to the level of $0.25 \mu \mathrm{rad}(\mathrm{rms})$. This is the performance level, characteristic for the world's best slope measuring instruments, such as the BESSY NOM. The major factors made for application of the advanced techniques are the 2D detector and the upgraded LTP translation system that is now capable of move-stop-wait-measuremove mode of operation combined with scanning in the forward and reverse directions.

We have illustrated the performance of the new version ALS LTP-II upgraded at the OML with measurements with super polished $\mathrm{x}$-optics fabricated for the MERLIN project. It was shown that the upgraded LTP is able to provide reliable slope measurements of significantly curved optics with slope variation as low as $0.25 \mu \mathrm{rad}(\mathrm{rms})$.

More work on improvement of the ALS LTP-II is still in progress.

We believe that the contribution of the systematic errors to the measurements would be significantly suppressed by appropriate calibration. A precise tilting stage that is a key element of such a calibration system is under customized fabrication at PI Gmbh \& Co.

Further improvement of random error associated with air convection in the LTP optical reference channel can be obtained with an additional reference channel based on a set of precise tiltmeters. The random noise due to the pointing instability of the LTP light beams can be significantly decreased with a fiber coupled laser diode system.

Improvement of the environmental conditions at the OML would help to decrease drift of the instrument with temperature. From the point of view of drift, it will be beneficial to replace the phase shifter based on roof prisms with a step phase shifter.

Our goal is to develop a slope measurement instrument with $\sim 0.1 \mu \mathrm{rad}$ performance, suitable for fully automatic operational mode. The instrument should allow a long term scanning sequence with reverse of scan direction, change of sagittal and tangential tilt, flip of orientation of the optics under test, etc. This would allow us to perform the measurements during night at the most stable environmental and background conditions at the OML, minimizing the instrumental noise and systematic errors.

\section{ACKNOWLEDGEMENTS}

The authors are grateful Rich Celestre, Rob Duarte, Keith D. Franck, Ralf Geckeler, Steve Irick, Alastair MacDowell, Howard Padmore, Artur Stepanyan, Peter Takacs, Tony Warwick, Thomas Zeschke, and Max Zolotorev for useful discussions and invaluable assistance. We wish to specifically mention and acknowledge our friend and colleague Frank Siewert for an enjoyable collaboration and constructive comments to the manuscript.

The Advanced Light Source is supported by the Director, Office of Science, Office of Basic Energy Sciences, Material Science Division, of the U.S. Department of Energy under Contract No. DE-AC02-05CH11231 at Lawrence Berkeley National Laboratory.

\section{DISCLAIMER}

This document was prepared as an account of work sponsored by the United States Government. While this document is believed to contain correct information, neither the United States Government nor any agency thereof, nor The Regents of the University of California, nor any of their employees, makes any warranty, express or implied, or assumes any legal responsibility for the accuracy, completeness, or usefulness of any information, apparatus, product, or process disclosed, or represents that its use would not infringe privately owned rights. Reference herein to any specific commercial product, process, or service by its trade name, trademark, manufacturer, or otherwise, does not necessarily constitute or imply its endorsement, recommendation, or favoring by the United States Government or any agency thereof, or The Regents of the University of California. The views and opinions of authors expressed herein do not necessarily state or reflect those of the United States Government or any agency thereof or The Regents of the University of California.

\section{REFERENCES}

[1] Yashchuk, V. V., "Positioning errors of pencil-beam interferometers for long-trace profilers," Proc. SPIE 6317, 63170A-12 (2006). 
[2] Kirschman, J. L., Smith, B. V., Domning, E. E., Irick, S. C., MacDowell, A. A., McKinney, W. R., Morrison, G. Y., Smith, B. V., Warwick, T., and Yashchuk, V. V., "Flat-Field Calibration of CCD Detector for Long Trace Profilers," Proc. SPIE 6317, 67040J-11 (2007).

[3] Kirschman, J. L., Domning, E. E., Morrison, G. Y., Smith, B. V., Yashchuk, V. V., "Precision Tiltmeter as a Reference for Slope Measuring Instruments," Proc. SPIE 6317, 670409-12 (2007).

[4] MERLIN (Milli-Electron-volt Resolution beamLINe) project developed by the ALS Scientific Support Group, Beamline 4.0.1: www-als.lbl.gov/als/techspecs/ b14.0.1.html.

[5] Siewert, F., Noll, T., Schlegel, T., Zeschke, T., and Lammert, H., "The Nanometre Optical Component Measuring Machine: a new Sub-nm Topography Measuring Device for X-ray Optics at BESSY," AIP Conf. Proc. 705, 847-850 (2004).

[6] Goldberg, K. and Yashchuk, V. V., "Advanced X-ray Optics Metrology for Nanofocusing and Coherence Preservation," Meeting Report in Synchrotron Radiation News 21(2), 11-12 (2008).

[7] Bray, M., "Stitching Interferometry: Recent results and Absolute calibration," Proc. SPIE 5252, 305-313 (2004).

[8] Church, E. L., Takacs, P. Z., "Use of an optical profiling instrument for the measurement of the figure and finish of optical quality surfaces," Wear 109 (1986), 241-57.

[9] Takacs, P. Z., Qian, Shinan, Colbert, J., "Design of a long trace surface profiler,” Proc. SPIE 749, 59-64 (1987).

[10] Takacs, P. Z., Feng, S. K., Church, E. L., Qian, Shinan, Liu, W-M., "Long trace profile measurements on cylindrical aspheres," Proc. SPIE 966, 354-64 (1989).

[11] Irick, S. C., McKinney, W. R., “Advancements in one-dimensional profiling with a long trace profiler,” Proc. SPIE, 1720, 162-168 (1992).

[12] Irick, S. C., McKinney, W. R., Lunt, D. L., Takacs, P. Z., "Using a straightness reference in obtaining more accurate surface profiles from a long trace profiler (for synchrotron optics)," Rev. Sci. Instrum. 63(1), 1436-8 (1992).

[13] Irick, S. C., "Improved measurement accuracy in a long trace profiler: compensation for laser pointing instability," Nucl. Instrum. \& Meth. in Phys. Res. A 347(1-3), 226-30 (1994).

[14] von Bieren K. "Pencil beam interferometer for aspherical optical surfaces," Proc. SPIE 343, 101-108 (1982).

[15] Qian, Shinan, Li, H., Takacs, P. Z., "Penta-Prism Long Trace Profiler (PPLTP) for measurement of grazing incidence space optics," Proc. SPIE, 2805, 108-114 (1996).

[16] Takacs, P.Z., Qian, Shinan, Kester, T., Li, H., "Large-mirror figure measurement by optical profilometry techniques," Proc. SPIE 3782, 266-274 (1999).

[17] Qian, Shinan, Sostero, G., Takacs, P. Z., "Precision calibration and systematic error reduction in the Long Trace Profiler," Proc. SPIE 3782, 627-636 (1999).

[18] Qian, Shinan, Sostero G., Takacs, P. Z., "Precision calibration and systematic error reduction in the long trace profiler," Opt. Engineering 39(1), 304-310 (2000).

[19] Takacs, P. Z., Qian, Shinan, “Accuracy limitations in long-trace profilometry,” AIP Conf. Proc. 708, 831-834 (2004).

[20] Qian, Shinan, Jark, W., Takacs, P. Z., "The penta-prism LTP: a long-trace-profiler with stationary optical head and moving penta prism," Rev. Sci. Instrum. 66(3), 2562-2569 (1995).

[21] Takacs, P. Z., Bresloff, C. J., "Significant improvements in long trace profiler measurement performance," Proc. SPIE 2856, 236-245 (1996).

[22] P. Z. Takacs, E. L. Church, C. J. Bresloff, Assoufid, L., "Improvements in the accuracy and the repeatability of long trace profiler measurements," Appl. Optics 38(25), 5468-5479 (1999).

[23] Qian, Shinan, Takacs, P.Z., "Equal optical path beamsplitter for a pencil beam interferometer and shearing interferometer," Opt. Engineering 42(4), 929-934 (2003).

[24] Yashchuk, V. V., Irick, S. C., MacDowell, A. A., "Elimination of 'ghost'-effect-related systematic errors in metrology of X-ray optics with a long trace profiler,” Proc. SPIE 5858, 58580X-8 (2005).

[25] Pawliszyn, J., Weber, M. F., Dignam, M. J., "Dual-beam laser deflection sensor," Rev. Sci. Instrum. 56(9), 17401743 (1985).

[26] Yashchuk, V. V., Irick, S. C., MacDowell, A. A., McKinney, W. R., Takacs, P. Z., “Air convection noise of pencilbeam interferometer for long-trace profiler," Proc. SPIE 6317, 63170D-12 (2006).

[27] Siewert, F. "Calibration: Autocalibration and Calibration," on 3rd International Workshop on Metrology for X-ray Optics, Daegu, Korea, June 2006.

[28] Yashchuk, V. V., McKinney, W. R., Warwick, T., Noll, T., Siewert, F., Zeschke, T., Geckeler, R. D., "Proposal for a Universal Test Mirror for Characterization of Slope Measuring Instruments,” Proc. SPIE 67040A-12(2007). 
[29] Yashchuk, V. V., "Universal Test Mirror for Calibration of Long Trace Profilers: Proposal," LSBL-800 (October, 2006).

[30] Yashchuk, V. V., "Discussing a Universal Test Mirror for Characterization of Slope Measuring Instruments," LSBL-861 (November, 2007)

[31] Yashchuk, V. V., "Performance Test of the Long Trace Profiler. Part 1: Random Noise Limit of Slope Measurement with Mirror M4 for BL 9.0.2," LSBL-710 (August, 2004).

[32] Irick, S. C., McKinney, W. R., Peterman, D., and Yashchuk, V. V., "Performance Test of the Long Trace Profiler. Part 5.1: Systematic Noise due to Laser Pointing Instability," LSBL-762 (October, 2005).

[33] Irick, S. C., MacDowell, A. A., McKinney, W. R., and Yashchuk, V. V., "Performance Test of the Long Trace Profiler. Part 2: Systematic Errors of Slope Measurement Related to the Performance of the LTP Photo-detector," LSBL-714 (September, 2004).

[34] Yashchuk, V. V., "Performance Test of the Long Trace Profiler. Part 4: Estimation of Laser Beam Positioning Error," LSBL-741 (March, 2005).

[35] Kirschman, J. L., Irick, S. C., McKinney, W. R., Yashchuk, V. V., "Metrology of U8G1 GLIDCOP Grating Blank for ALS Beamline 10.0.1," LSBL-842 (May, 2007).

[36] Kirschman, J. L., McKinney, W. R., Yashchuk, V. V., "Upgrade of the Long Trace Profiler LTP-II. Part 3: A New Method for Suppression of the LTP Systematic Error," LSBL-829 (June, 2007).

[37] Kirschman, J. L., Celestre, R. S., Irick, S. C., Warwick, T., Yashchuk, V. V., "Temperature Stability in the Optical Metrology Laboratory,” LSBL-793 (July, 2006).

[38] Yashchuk, V. V., "Optical Metrology: Why It Matters to You?” LSBL-850 (October, 2007).

[39] Melles Griot Inc. (http://lasers.mellesgriot.com); 57 ICS-Series Fiber-Coupled Diode Lasers Module.

[40] Photon Inc. (http://photon-inc.com); NanoScan High-Power Sub-Micron Accuracy Beam Profiler.

[41] Qian, Shinan and Takacs, P. Z., "Wave front-splitting phase shift beam splitter for pencil beam interferometer," Rev. Sci. Instrum. 74(11), 4881-4884 (2003).

[42] Kavaldjiev, D. and Ninkov, Z., "Subpixel sensitivity map for a charge-coupled device sensor," Opt. Engineering 37(3), 948-954 (1998).

[43] Domning, E. E., Franck, K. D., Kirschman, J. L., MacDowell, A. A.., McKinney, W. R., Morrison, G. Y., Smith, B. V., Warwick, T., Yashchuk, V. V., "Upgrade of the Long Trace Profiler LTP-II. Part 1: Upgrade of the Long Trace Profiler LTP-II. Part 1: Modification and Tuning of the LTP-II Translation System," LSBL-832 (April, 2007).

[44] Domning, K. D., Kirschman, J. L., MacDowell, A. A.., McKinney, W. R., Morrison, G. Y., Smith, B. V., Warwick, T., Yashchuk, V. V., "Upgrade of the Long Trace Profiler LTP-II. Part 2: New 2D CCD Detector and Data Acquisition System," LSBL-874 (March, 2008).

[45] Kirschman, J. L., McKinney, Yashchuk, V. V., "Upgrade of the Long Trace Profiler LTP-II. Part 4: LTP Metrology of 18.7-m Grating Substrate for the MERLIN Project," LSBL-830 (April, 2007).

[46] F. Polack (private communication).

[47] Pantera SA 4M15 Area-Scan Camera; http://mv.dalsa.com.

[48] Yashchuk, V. V., Franck, A. D., Irick, S. C., Howells, M. R., MacDowell, A. A., W. R. McKinney, "Two dimensional power spectral density measurements of X-ray optics with the Micromap interferometric microscope," Proc. SPIE 5858, 58580A-12 (2005).

[49] Yashchuk, V. V., Gullikson, E. M., Howells, M. R., Irick, S. C., MacDowell, A. A., McKinney, W. R., Salmassi, F., Warwick, T., Metz, J. P., and Tonnessen, T. W., "Surface Roughness of Stainless Steel Mirrors for Focusing Soft Xrays," Appl. Optics 45(20) 4833-4842 (2006).

[50] Kirschman, J. L., McKinney, W. R., Yashchuk, V. V., "Metrology of $152 \mathrm{~mm}$ silicon spherical grating blank G301 for ALS BL 4.0.3 (MERLIN)," LSBL-888 (December, 2007).

[51] Siewert, F., "Metrology of Water Cooled Spherical Grating Blank of the MERLIN-Beamline at the Advanced Light Source," BESSY Measurement Report (July, 21007).

[52] Kirschman, J. L., McKinney, W. R., Yashchuk, V. V., "Operational Manual for the Upgraded ALS LTP-II," LSBL Note (in preparation).

[53] McKinney, W. R., Irick, S. C., "XUV synchrotron optical components for the Advanced Light Source: summary of the requirements and the developmental program," Proc. SPIE 1740, 154-160 (1993). 